\title{
INVESTIGATION OF SPATIAL QUASISTATIC BENDING OF REINFORCED CONCRETE FRAMES TAKING INTO ACCOUNT PHYSICAL NONLINEARITY AND PLASTICITY OF CONCRETE BY THE BOUNDARY ELEMENT METHOD
}

\author{
${ }^{1}$ Fomin V. M., Doctor of Science, Professor, \\ vmfomin39@gmail.com, ORCID: 0000-0003-4814-6415 \\ ${ }^{1}$ Fomina I. P., Senior lecturer, \\ fomina@ogasa.org.ua, ORCID: 0000-0001-7366-1537 \\ ${ }^{1}$ Odessa State Academy of Civil Engineering and Architecture \\ 4, Didrikhson street, Odessa, 65029, Ukraine
}

\begin{abstract}
Implementation of a direct dynamic calculation of spatial reinforced concrete frame structures taking into account the nonlinear work and plastic properties of materials is rather a difficult task. When carrying out such a calculation by the finite element method, difficulties arise at the computational stage and in analysis of the results due to the fact that the dimension of the system of equations of this method is very large, since it is necessary to split the elements of reinforced concrete frames into a large number of small finite elements due to the difference in deformation properties of concrete in compressed and stretched zones, as well as in the stages of loading and unloading.

In connection with the stated before remarks, there is a need for the use of alternative methods, especially in the study of multi-cycle dynamic processes, for example, with seismic effects.

On the base of the method for constructing differential equations of spatial non-linear elastoplastic bending of reinforced concrete beams and the algorithm for applying the boundary element method in solving non-linear problems proposed by the author, an algorithm for solving quasistatic problems for reinforced concrete frames is developed.

On each of the rods, a local coordinate system is selected. In this system, for each of the rods, a system of differential equations of spatial bending is written in increments of the coordinates of the cross section center and the torsion angle, taking into account the physical and geometric nonlinearities and plasticity of concrete. On the base of the algorithm proposed by the author, by means of fundamental solutions to the Cauchy problem for this system, matrices can be constructed for each rod, by means of which vectors of increments of displacements, bending moments and transverse forces in each of the cross sections of the rod can be found.

The systems of matrix equations defining these relations can be written for the entire frame in the form of a single equation.

On the base of solving this equation, the increments of the stresses in the frame rods and changes of their curved axes are determined at each calculation step.

Keywords: boundary element method, reinforced concrete beams and frames, spatial bending, physical nonlinearity and plasticity of concrete.

Introduction. In recent decades, direct calculation (i.e., without the use of simplified models) of structures on dynamic, in particular, seismic, influences (for seismic - specified in the form of accelerograms of earthquakes) has become widespread.

However, it turned out that the direct dynamic calculation of spatial structures, taking into account the nonlinear work and plastic properties of materials is rather a difficult task. When carrying out such a calculation by the finite element method, difficulties arise at the computational stage and in the analysis of the results.

Analysis of recent research. This is because the dimension of the equation system of this method is very large. Because it is necessary to split the elements of reinforced concrete frames into a large number of small finite elements. In these elements, the deformation properties of concrete in the compressed and stretched zones differ significantly. They also differ in loading and unloading stages $[1-5,6]$.
\end{abstract}


There was a need for the construction of alternative methods with respect to the finite element method, especially when studying multi-cycle dynamic processes, for example, under seismic influences.

One of such alternative methods is the boundary element method.

Objectives and problems. To use this method in the study of nonlinear elastoplastic problems of spatial bending of reinforced concrete beams using the step-by-step method, it is necessary to solve the following problems:

1. Construction of a system of spatial bending differential equations of reinforced concrete beams forming the considered reinforced concrete frame, taking into account the physical nonlinearity and plasticity of concrete.

2. Construction of matrix $\boldsymbol{A}(s)$ of fundamental solutions to the Cauchy problem for a homogeneous system of differential equations corresponding to the constructed system of differential equations for each of the frame beams.

3. Construction of a particular solution $\boldsymbol{d} \boldsymbol{w}_{p}(s)$ of the constructed inhomogeneous system of differential equations.

4. Construction of an algebraic equations resolving system for determining support reactions and coefficients of formulas for determining internal forces and deformations in the frame rods.

Materials and research methods. We consider the spatial bending of the $\Pi$-shaped frame (Fig. 1), consisting of three reinforced concrete rods 1, 2 and 3 (the rod numbers are shown in parentheses) of constant rectangular cross section, rods 1 and 3 are rigidly clamped at points $\mathrm{A}$ and $\mathrm{B}$.

A local coordinate system is selected on each of the rods $x_{1}^{(i)}, x_{2}^{(i)}, x_{3}^{(i)}$ (i number of the rod). The drawing also shows the global coordinate system $x_{1}, x_{2}, x_{3}$. For nodes 1 and 2 (node numbers in square brackets), the forces $\boldsymbol{P}_{j}^{[1]}(j=1,2,3)$ and $\boldsymbol{P}_{j}^{[2]}(j=1,2)$, parallel to the axes of the global coordinate system, are applied. The drawing also shows the components of the support reactions $R_{A}$ and $R_{B}$ and the support moments of $M_{A}$ and $M_{B}$.

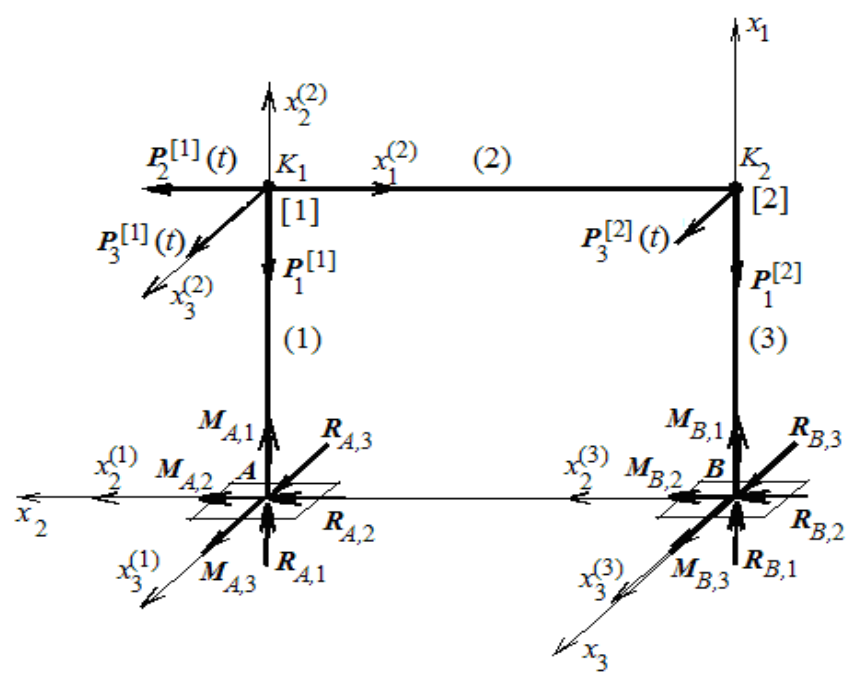

Fig. 1. Frame scheme

The system of differential equations for the spatial bending of a reinforced concrete beam in increments of the coordinate center of the cross section and the torsion angle, taking into account the physical and geometric nonlinearities and plasticity of concrete, has the following form [6]:

$$
\begin{aligned}
& X_{i, 1}(s) d x_{2}^{\prime \prime \prime}-X_{i, 2}(s) d x_{3}^{\prime \prime \prime}+X_{i, 3}(s) d \theta^{\prime \prime}+Y_{i, 1}(s) d x_{2}^{\prime \prime}-Y_{i, 2}(s) d x_{3}^{\prime \prime}+ \\
& +Y_{i, 3}(s) d \theta^{\prime}+Z_{i, 1}(s) d x_{2}^{\prime}-Z_{i, 2}(s) d x_{3}^{\prime}+Z_{i, 3}(s) d \theta+\sum_{k=1}^{3} \tilde{U}_{i, k}(s) d F_{k}=0 \quad(i=1,2,3),
\end{aligned}
$$


and the boundary conditions are as follows:

$$
\begin{aligned}
& V_{i, 1} d x_{2}^{\prime}(0)-V_{i, 2} d x_{3}^{\prime}(0)+V_{3} d \theta(0)+V_{i, 4} d x_{2}^{\prime \prime}(0)-V_{i, 5} d x_{3}^{\prime \prime}(0)+V_{i, 6} d \theta^{\prime}(0)+ \\
& +\sum_{k=1}^{3} W_{i, k} d F_{k}+d M_{0, i}=0(i=1,2,3) .
\end{aligned}
$$

Dash means derivative with respect to the arc coordinate $s, d F_{i}$ and $d M_{o, i}$ are components of the increment of the total vector $\boldsymbol{d} F$ and the total moment $\boldsymbol{d} \boldsymbol{M}_{o}$ of external forces applied to the left end of the beam; the coefficients in expressions (2) and (3) are determined at the previous step of the step-by-step method. We write (2) as follows:

$$
\begin{aligned}
& \boldsymbol{B} d \eta(0)+\boldsymbol{C} d \eta^{\prime}(0)=-\boldsymbol{W} d \boldsymbol{F}-d \boldsymbol{M}_{o}, \\
& \boldsymbol{B}=\left[\begin{array}{cc}
V_{1,1}-V_{1,2} & V_{1,3} \\
V_{2,1}-V_{2,2} & V_{2,3} \\
V_{3,1}-V_{3,2} & V_{3,3}
\end{array}\right], \boldsymbol{C}=\left[\begin{array}{cc}
V_{1,4}-V_{1,5} & V_{1,6} \\
V_{2,4}-V_{2,5} & V_{2,6} \\
V_{3,4}-V_{3,5} & V_{3,6}
\end{array}\right], \boldsymbol{d} \eta=\left[\begin{array}{c}
d x_{C, 2}^{\prime}(s) \\
d x_{C, 3}^{\prime}(s) \\
d \theta(s)
\end{array}\right] .
\end{aligned}
$$

From (3) we obtain:

$$
d \eta^{\prime}(0)=\boldsymbol{D} d \eta(0)+\boldsymbol{E} d \boldsymbol{M}_{o}+\boldsymbol{G} d \boldsymbol{F},
$$

where $\boldsymbol{D}=-\boldsymbol{C}^{-1} \boldsymbol{B}, \boldsymbol{E}=-\boldsymbol{C}^{-1}, \boldsymbol{G}=-\boldsymbol{C}^{-1} \boldsymbol{W}$. Let us introduce a vector $d \boldsymbol{w}(s)$ with elements:

$$
\begin{aligned}
& d w_{1}(s)=d x_{2}(s), d w_{2}(s)=d x_{3}(s), d w_{3}(s)=d x_{2}^{\prime}(s), d w_{4}(s)=d x_{3}^{\prime}(s), \\
& d w_{5}(s)=d \theta(s), d w_{6}(s)=d x_{2}^{\prime \prime}(s), d w_{7}(s)=d x_{3}^{\prime \prime}(s), d w_{8}(s)=d \theta^{\prime}(s), d w_{9}(s)=d x_{1}(s)
\end{aligned}
$$

Then (4) can be written as follows:

$$
d \boldsymbol{w}(0)=\tilde{\boldsymbol{D}} d \tilde{\boldsymbol{w}}(0)+\tilde{\boldsymbol{E}} d \boldsymbol{M}_{o}+\tilde{\boldsymbol{G}} d \boldsymbol{F},
$$

where $d \tilde{\boldsymbol{w}}(0)$ is a vector $d \boldsymbol{w}(0)$, at that $d w_{j}(0)=0(j=6,7,8,9), \tilde{\boldsymbol{D}}$ is a ninth-order square matrix in which $\widetilde{\boldsymbol{D}}_{i i}=1(i=1,2, \ldots, 5), \widetilde{\boldsymbol{D}}_{i j}=\boldsymbol{D}_{i-5, j-2}(i=6,7,8 ; j=3,4,5), \widetilde{\boldsymbol{D}}_{9,9}=11, \tilde{\boldsymbol{E}}$ and $\tilde{\boldsymbol{G}}$ are matrices of $9 \times 3$ order in which $\widetilde{\boldsymbol{E}}_{i, j}=\boldsymbol{E}_{i-5, j}, \widetilde{\boldsymbol{G}}_{i, j}=\boldsymbol{G}_{i-5, j}(i=6,7.8 ; j=6,7,8)$. The remaining elements of the matrices $\tilde{\boldsymbol{D}}, \tilde{\boldsymbol{E}}$ and $\tilde{\boldsymbol{G}}$ are equal to zero.

For the rod $1 d \boldsymbol{F}=d \boldsymbol{R}_{A}, d \boldsymbol{M}_{o}=d \boldsymbol{M}_{A}$ and equality (6) looks like this:

$$
d \boldsymbol{w}(0)=\tilde{\boldsymbol{D}} d \widetilde{\boldsymbol{w}}(0)+\widetilde{\boldsymbol{E}} d \boldsymbol{M}_{A}+\widetilde{\boldsymbol{G}} d \boldsymbol{R}_{A} .
$$

Based on the algorithm proposed in [7], for each rod the matrix $\boldsymbol{A}(s)$ and the vector $\boldsymbol{d} \boldsymbol{w}_{\boldsymbol{u}}(s)$ can be constructed, and with their help a vector can be found:

$$
d \boldsymbol{w}(s)=\boldsymbol{A}(s) d \boldsymbol{w}(0)+\boldsymbol{d} \boldsymbol{w}_{\boldsymbol{u}}(s),
$$

$A_{1, j}=z_{1, j}(s), A_{2, j}=z_{1, j}^{\prime}(s), A_{3, j}=z_{1, j}^{\prime \prime}(s), A_{4, j}=z_{2, j}(s), A_{5, j}=z_{2, j}^{\prime}(s), A_{6, j}=z_{2, j}^{\prime \prime}(s), A_{7, j}=$ $=z_{3, j}(s), A_{8, j}=z_{1, j}^{\prime}(s)(j=1,2, \ldots, 8) . \quad\left(z_{k, j}(s)(k=1,2,3)-\right.$ are fundamental solutions to the Cauchy problem for the system of differential equations (1)), $A_{9,9}=1$, the remaining elements of the ninth row and the ninth column of the matrix $\boldsymbol{A}$ are zero, and $d \boldsymbol{w}_{u}^{(1)}(s)$ is determined by the formula:

$$
d \boldsymbol{w}_{u}^{(1)}(s)=\boldsymbol{S}(s) d \boldsymbol{F}
$$

$S(s)$ is a matrix with elements $z_{u, j, m}(s)(j=1,2, \ldots, 8 ; m=1,2,3)$ [7], the remaining elements of the matrix $S(s)$ are equal to zero. Note that due to the small deflections of the reinforced concrete beams, we can put $s=x_{1}^{(j)}$ ( $j$ is a frame rod number).

For the rod 1:

$$
d \boldsymbol{w}_{p}^{(1)}(s)=\boldsymbol{S}^{(1)}(s) d \boldsymbol{R}_{A}
$$


Given that $d x_{1}(0)=d x_{2}(0)=d x_{2}^{\prime}(0)=d x_{3}(0)=d x_{3}^{\prime}(0)=d \theta(0)=0$ (т.e. $\left.d \widetilde{\boldsymbol{w}}^{(1)}(0)=0\right)$, we find from $(8)$ :

$$
d \boldsymbol{w}^{(1)}(0)=\boldsymbol{w}_{M}^{(1)}(0) d \boldsymbol{M}_{A}+w_{R}^{(1)}(0) d \boldsymbol{R}_{A},
$$

where $\boldsymbol{w}_{M}^{(1)}(0)=\widetilde{\boldsymbol{E}}^{(1)}, \boldsymbol{w}_{R}^{(1)}(0)=\widetilde{\widetilde{\boldsymbol{G}}}^{(1)}$. It follows from (9) - (11) that:

$$
d \boldsymbol{w}^{(1)}(s)=\boldsymbol{w}_{M}^{(1)}(s) d \boldsymbol{M}_{A}+\boldsymbol{w}_{R}^{(1)}(s) d \boldsymbol{R}_{A},
$$

where $\boldsymbol{w}_{M}^{(1)}(s)=\boldsymbol{A}^{(1)}(s) \tilde{\boldsymbol{E}}^{(1)}, \boldsymbol{w}_{R}^{(1)}(s)=\boldsymbol{A}^{(1)}(s) \tilde{\boldsymbol{G}}^{(1)}+\boldsymbol{S}^{(1)}(s)$. For $s=l_{1}$, in accordance with (5), we obtain the values $d x_{2}\left(l_{1}\right), d x_{2}^{\prime}\left(l_{1}\right), d x_{2}^{\prime \prime}\left(l_{1}\right), d x_{3}\left(l_{1}\right), d x_{3}^{\prime}\left(l_{1}\right), d x_{3}^{\prime \prime}\left(l_{1}\right), d \theta\left(l_{1}\right), d \theta^{\prime}\left(l_{1}\right)$.

To find $d M_{i}(s)$ and $d Q_{i}(s)(i=1,2,3)$ we use formulas (21) [8]:

$$
\begin{aligned}
& \left.d M_{i}(s)=\left(\tilde{M}_{i}\right)_{1}(s) d x_{2}^{\prime}(s)-\left(\tilde{M}_{i}\right)_{2} s\right) d x_{3}^{\prime}(s)+\left(\tilde{M}_{i}\right)_{3}(s) d \theta(s)+ \\
& +\left(\tilde{M}_{i}\right)_{4}(s) d x_{2}^{\prime \prime}(s)-\left(\tilde{M}_{i}\right)_{5}(s) d x_{3}^{\prime \prime}(s)+\left(\tilde{M}_{i}\right)_{6}(s) d \theta^{\prime}(s)+\sum_{m=1}^{3}\left(\tilde{\tilde{M}}_{i}\right)_{m}(s) d F_{m}(i=1,2,3), \\
& d Q_{i}(s)=\left(\tilde{Q}_{i}\right)_{3}(s) d x_{2}^{\prime}(s)-\left(\tilde{Q}_{i}\right)_{2}(s) d x_{3}^{\prime}(s)+\left(\tilde{Q}_{i}\right)_{3}(s) d \theta(s)+ \\
& +\left(\tilde{Q}_{i}\right)_{4}(s) d x_{2}^{\prime \prime}(s)-\left(\tilde{Q}_{i}\right)_{5}(s) d x_{3}^{\prime \prime}(s)+\left(\tilde{Q}_{i}\right)_{6}(s) d \theta^{\prime}(s)+\sum_{m=1}^{3}\left(\tilde{\widetilde{Q}}_{i}\right)_{m}(s) d F_{m}(i=1,2,3),
\end{aligned}
$$

(here designations $\left(\tilde{M}_{i}\right)_{r}=\left(M_{i}\right)_{r}^{(3)},\left(\tilde{Q}_{i}\right)_{r}=\left(Q_{i}\right)_{r}^{(3)},\left(\tilde{\tilde{M}}_{i}\right)_{r}=\left(M_{i}\right)_{r}^{(4)},\left(\tilde{\widetilde{Q}}_{i}\right)_{r}=\left(Q_{i}\right)_{r}^{(4)}$ are introduced).

Formulas (13) can be written as follows:

Here:

$$
d \boldsymbol{M}(s)=\tilde{\boldsymbol{M}}(s) d \boldsymbol{w}(s)+\tilde{\widetilde{\boldsymbol{M}}}(s) d \boldsymbol{F}, d \boldsymbol{Q}(s)=\tilde{\boldsymbol{Q}}(s) d \boldsymbol{w}(s)+\tilde{\widetilde{\boldsymbol{Q}}}(s) d \boldsymbol{F}
$$

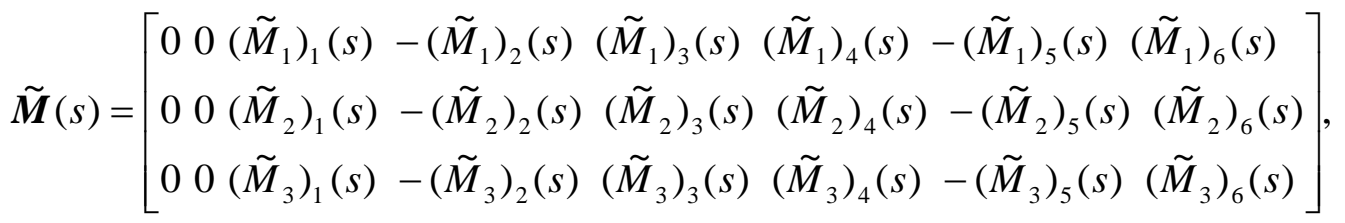

$$
\begin{aligned}
& \tilde{\tilde{M}}(s)=\left[\begin{array}{ccc}
\left(\tilde{\tilde{M}}_{1}\right)_{1}(s) & \left(\tilde{\tilde{M}}_{1}\right)_{2}(s) & \left(\tilde{\tilde{M}}_{1}\right)_{3}(s) \\
\left(\tilde{\tilde{M}}_{2}\right)_{1}(s) & \left(\tilde{\tilde{M}}_{2}\right)_{2}(s) & \left(\tilde{\tilde{M}}_{2}\right)_{3}(s) \\
\left(\tilde{\tilde{M}}_{3}\right)_{1}(s) & \left(\tilde{\tilde{M}}_{3}\right)_{2}(s) & \left(\tilde{\tilde{M}}_{3}\right)_{3}(s)
\end{array}\right] .
\end{aligned}
$$

Matrices $\tilde{\boldsymbol{Q}}(s)$ and $\tilde{\widetilde{\boldsymbol{Q}}}(s)$ have a similar form.

For rod 1, taking into account (12), we obtain:

$$
\begin{aligned}
& d \boldsymbol{M}^{(1)}(s)=\tilde{\boldsymbol{M}}^{(1)}(s) \boldsymbol{w}_{M}^{(1)}(s) d \boldsymbol{M}_{A}+\left[\tilde{\boldsymbol{M}}^{(1)}(s) \boldsymbol{w}_{R}^{(1)}(s)+\tilde{\widetilde{\boldsymbol{M}}}^{(1)}(s)\right] d \boldsymbol{R}_{A}, \\
& d \boldsymbol{Q}^{(1)}(s)=\tilde{\boldsymbol{Q}}^{(1)}(s) \boldsymbol{w}_{M}^{(1)}(s) d \boldsymbol{M}_{A}+\left[\tilde{\boldsymbol{Q}}^{(1)}(s) \boldsymbol{w}_{R}^{(1)}(s)+\tilde{\widetilde{\boldsymbol{Q}}}^{(1)}(s)\right] d \boldsymbol{R}_{A},
\end{aligned}
$$

what can be written the next way:

$$
\begin{aligned}
& d \boldsymbol{M}^{(1)}(s)=\boldsymbol{M}_{M}^{(1)}(s) d \boldsymbol{M}_{A}+\boldsymbol{M}_{R}^{(1)}(s) d \boldsymbol{R}_{A}, \\
& d \boldsymbol{Q}^{(1)}(s)=\boldsymbol{Q}_{M}^{(1)}(s) d \boldsymbol{M}_{A}+\boldsymbol{Q}_{R}^{(1)}(s) d \boldsymbol{R}_{A},
\end{aligned}
$$

where $\boldsymbol{M}_{M}^{(1)}(s)=\tilde{\boldsymbol{M}}^{(1)}(s) \boldsymbol{w}_{M}^{(1)}(s), \boldsymbol{M}_{R}^{(1)}(s)=\tilde{\boldsymbol{M}}^{(1)}(s) \boldsymbol{w}_{R}^{(1)}(s)+\widetilde{\tilde{\boldsymbol{M}}}^{(1)}(s), \boldsymbol{Q}_{M}^{(1)}(s)=\widetilde{\boldsymbol{Q}}^{(1)}(s) \boldsymbol{w}_{M}^{(1)}(s)$, $\boldsymbol{Q}_{R}^{(1)}(s)=\widetilde{\boldsymbol{Q}}^{(1)}(s) \boldsymbol{w}_{R}^{(1)}(s)+\tilde{\widetilde{\boldsymbol{Q}}}^{(1)}(s)$. Consider the part of the frame located to the left of the left end of the rod 2 (Fig. 2). Here $d \boldsymbol{F}^{(2)}$ and $d \boldsymbol{M}_{o}^{(2)}$ are increments of the total vector and the total moment of forces shown in the drawing. Define the projection $\boldsymbol{d \boldsymbol { F } ^ { ( 2 ) }}$ and $d \boldsymbol{M}_{O}^{(2)}$ on axes $x_{i}^{(2)}(i=1,2,3)$ : 


$$
\begin{aligned}
& d F_{1}^{(2,2)}=-d R_{A, 2}-d P_{2}^{[1]}, d F_{2}^{(2,2)}=d R_{A, 1}+d P_{1}^{[1]}, d F_{3}^{[1]}=d R_{A, 3}+d P_{3}^{[1]}, \\
& d M_{o, 1}^{(2,2)}=-d M_{A, 2}-d R_{A, 3} l_{1}, d M_{o, 2}^{(2,2)}=d M_{A, 1}, d M_{o, 3}^{[1]}=d M_{A, 3}-d R_{A, 2} l_{1} .
\end{aligned}
$$

These equalities can be written as follows:

$$
\begin{gathered}
d \boldsymbol{F}^{(2)}=\boldsymbol{K}^{[1]} d \boldsymbol{R}_{A}+\boldsymbol{K}^{[1]} d \boldsymbol{P}^{[1]}, d \boldsymbol{M}_{o}^{(2)}=\boldsymbol{K}^{[1]} d \boldsymbol{M}_{A}+\boldsymbol{L}^{[1]} d \boldsymbol{R}_{A}, \\
\boldsymbol{K}^{[1]}=\left[\begin{array}{lrr}
0 & -1 & 0 \\
1 & 0 & 0 \\
0 & 0 & 1
\end{array}\right], \boldsymbol{L}^{[1]}=l_{1}\left[\begin{array}{llr}
0 & 0 & -1 \\
0 & 0 & 0 \\
0 & -1 & 0
\end{array}\right] .
\end{gathered}
$$

For reinforced concrete structures, the Krylov angles are very small, so we can assume that in the local coordinate system, the angle $\theta$ is the angle of rotation around the axis $x_{1}, \psi-$ around the $x_{2}$,

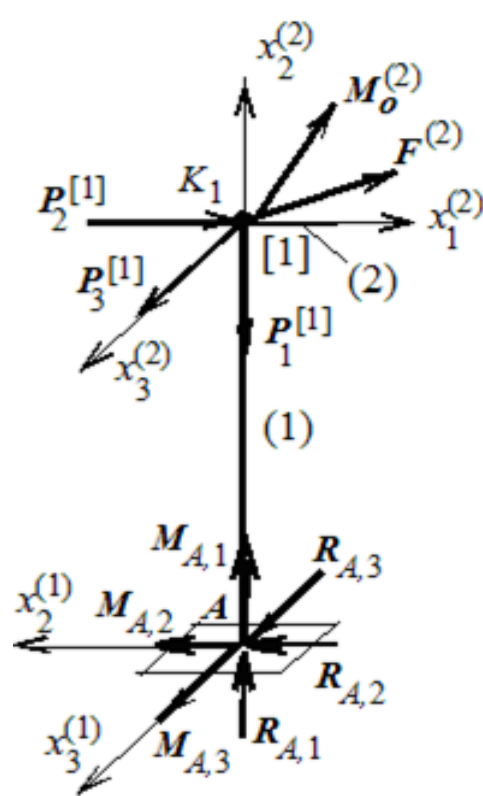

Fig. 2. System of external forces applied to the rod 1 $\varphi$ - around the $x_{3}$. Directions of the axes $x_{2}^{(2)}$ and $x_{1}^{(1)}$, as well as axes $x_{3}^{(2)}$ and $x_{3}^{(1)}$ coincide, but directions of the axes $x_{1}^{(2)}$ and $x_{2}^{(1)}$ are directly opposite. From here it follows that:

$d \phi^{(2)}(0)=d \phi^{(1)}\left(l_{1}\right), d \psi^{(2)}(0)=d \theta^{(1)}\left(l_{1}\right), d \theta^{(2)}(0)=-d \psi^{(1)}\left(l_{1}\right)$.

From these relations and from the fact that $d x_{C, 2}^{\prime}=d \phi, d x_{C, 3}^{\prime}=-d \psi$ [7], we get:

$$
\begin{aligned}
& d w_{1}^{(2)}(0)=d x_{2}^{(2)}(0)=d x_{1}^{(1)}\left(l_{1}\right)=d w_{9}^{(1)}\left(l_{1}\right), \\
& d w_{2}^{(2)}(0)=d x_{3}^{(2)}(0)=d x_{3}^{(1)}\left(l_{1}\right)=d w_{2}^{(1)}\left(l_{1}\right), \\
& d w_{3}^{(2)}(0)=\left(d x_{2}^{(2)}\right)^{\prime}(0)=d \phi^{(2)}(0)=d \phi^{(1)}\left(l_{1}\right)=\left(d x_{2}^{(1)}\right)^{\prime}\left(l_{1}\right)=d w_{3}^{(1)}\left(l_{1}\right), \\
& d w_{4}^{(2)}(0)=\left(d x_{3}^{(2)}\right)^{\prime}(0)=-d \psi^{(2)}(0)=-d \theta^{(1)}\left(l_{1}\right)=-d w_{5}^{(1)}\left(l_{1}\right), \\
& d w_{5}^{(2)}(0)=d \theta^{(2)}(0)=-d \psi^{(1)}\left(l_{1}\right)=\left(d x_{3}^{(1)}\right)^{\prime}\left(l_{1}\right)=d w_{4}^{(1)}\left(l_{1}\right), \\
& d w_{9}^{(2)}(0)=d x_{1}^{(2)}(0)=-d x_{2}^{(1)}\left(l_{1}\right)=-d w_{1}^{(1)}\left(l_{1}\right) .
\end{aligned}
$$

Quantities $d w_{6}^{(2)}(0), d w_{7}^{(2)}(0), d w_{8}^{(2)}(0)$ remain undefined for now.

Let us write the relations (19) in matrix form:

$$
d \widetilde{\boldsymbol{w}}^{(2)}(0)=\boldsymbol{T}^{[1]} d \boldsymbol{w}^{(1)}\left(l_{1}\right),
$$

where $\boldsymbol{T}^{[1]}$ is a ninth-order square matrix in which

$$
\boldsymbol{T}_{1,9}^{[1]}=\boldsymbol{T}_{2,2}^{[1]}=\boldsymbol{T}_{3,3}^{[1]}=\boldsymbol{T}_{5,1}^{[1]}=1 ; \boldsymbol{T}_{4,4}^{[1]}=\mathbf{T}_{9,1}^{[1]}=-1,
$$

other elements are equal to zero. Let us use relation (6) for rod 2:

$$
d \boldsymbol{w}^{(2)}(0)=\tilde{\boldsymbol{D}}(0) d \tilde{\boldsymbol{w}}^{(2)}(0)+\widetilde{\boldsymbol{E}}(0) d \boldsymbol{M}_{o}^{(2)}+\tilde{\boldsymbol{G}}(0) d \boldsymbol{F}^{(2)} .
$$

From (19), (20) and (16) we obtain:

$$
d \boldsymbol{w}^{(2)}(0)=\boldsymbol{w}_{M}^{(2)}(0) d \boldsymbol{M}_{A}+\boldsymbol{w}_{R}^{(2)}(0) d \boldsymbol{R}_{A}+\boldsymbol{w}_{P}^{(2)}(0) d \boldsymbol{P}^{[1]} .
$$

Here $\boldsymbol{w}_{M}^{(2)}(0)=\widetilde{\boldsymbol{D}}(0) \boldsymbol{T}^{[1]} \boldsymbol{w}_{M}^{(1)}\left(l_{1}\right)+\widetilde{\boldsymbol{E}}(0) \boldsymbol{K}^{[1]}, \boldsymbol{w}_{R}^{(2)}(0)=\tilde{\boldsymbol{D}}(0) \boldsymbol{T}^{[1]} \boldsymbol{w}_{R}^{(1)}\left(l_{1}\right)+\widetilde{\boldsymbol{E}}(0) \boldsymbol{L}^{[1]}+\widetilde{\boldsymbol{G}}(0) \boldsymbol{K}^{[1]}$, $\boldsymbol{w}_{P}^{(2)}(0)=\tilde{\boldsymbol{G}}(0) \boldsymbol{K}^{[1]}$.

From (8) and (9) we find:

$$
d \boldsymbol{w}^{(2)}(s)=\boldsymbol{A}^{(2)}(s) d \boldsymbol{w}^{(2)}(0)+\boldsymbol{S}^{(2)}(s) d \boldsymbol{F}^{(2)} .
$$

Taking into account (24) and (21) we bring this equality to the following form:

$$
d \boldsymbol{w}^{(2)}(s)=\boldsymbol{w}_{M}^{(2)}(s) d \boldsymbol{M}_{A}+\boldsymbol{w}_{R}^{(2)}(s) d \boldsymbol{R}_{A}+\boldsymbol{w}_{P}^{(2)}(s) d \boldsymbol{P}^{[1]},
$$

where $\boldsymbol{w}_{M}^{(2)}(s)=\boldsymbol{A}^{(2)}(s) \boldsymbol{w}_{M}^{(2)}(0), \boldsymbol{w}_{R}^{(2)}(s)=\boldsymbol{A}^{(2)}(s) \boldsymbol{w}_{R}^{(2)}(0)+\boldsymbol{S}^{(2)}(s) \boldsymbol{K}^{[1]}, \boldsymbol{w}_{P}^{(2)}(s)=\boldsymbol{A}^{(2)}(s) \boldsymbol{w}_{P}^{(2)}(0)+$ $+\boldsymbol{S}^{(2)}(s) \boldsymbol{K}^{[1]}$.

It follows for the rod 2 from formulas (14):

$$
d \boldsymbol{M}^{(2)}(s)=\boldsymbol{M}_{M}^{(2)}(s) d \boldsymbol{M}_{A}+\boldsymbol{M}_{R}^{(2)}(s) d \boldsymbol{R}_{A}+\boldsymbol{M}_{P}^{(2)}(s) d \boldsymbol{P}^{[1]},
$$




$$
\begin{aligned}
& \boldsymbol{M}_{M}^{(2)}(s)=\tilde{\boldsymbol{M}}^{(2)}(s) \boldsymbol{w}_{M}^{(2)}(s), \boldsymbol{M}_{R}^{(2)}(s)=\tilde{\boldsymbol{M}}^{(2)}(s) \boldsymbol{w}_{R}^{(2)}(s)+\tilde{\tilde{\boldsymbol{M}}}^{(2)}(s) \boldsymbol{K}^{[1]}, \boldsymbol{M}_{P}^{(2)}(s)= \\
& =\tilde{\boldsymbol{M}}^{(2)}(s) \boldsymbol{w}_{P}^{(2)}(s)+\tilde{\tilde{\boldsymbol{M}}}^{(2)}(s) \boldsymbol{K}^{[1]} .
\end{aligned}
$$

Similarly, we obtain:

$$
d \boldsymbol{Q}^{(2)}(s)=\boldsymbol{Q}_{M}^{(2)}(s) d \boldsymbol{M}_{A}+\boldsymbol{Q}_{R}^{(2)}(s) d \boldsymbol{R}_{A}+\boldsymbol{Q}_{P}^{(2)}(s) d \boldsymbol{P}^{[1]} .
$$

Here $\boldsymbol{Q}_{M}^{(2)}(s)=\widetilde{\boldsymbol{Q}}^{(2)}(s) \boldsymbol{w}_{M}^{(2)}(s)+\widetilde{\widetilde{\boldsymbol{Q}}}^{(2)}(s) \boldsymbol{K}^{[1]}, \boldsymbol{M}_{R}^{(2)}(s)=\widetilde{\boldsymbol{Q}}^{(2)}(s) \boldsymbol{w}_{R}^{(2)}(s)+\tilde{\widetilde{\boldsymbol{Q}}}^{(2)}(s) \boldsymbol{K}^{[1]}, \boldsymbol{M}_{P}^{(2)}(s)=$ $=\widetilde{\boldsymbol{Q}}^{(2)}(s) \boldsymbol{w}_{P}^{(2)}(s)+\widetilde{\widetilde{\boldsymbol{Q}}}^{(2)}(s) \boldsymbol{K}^{[1]}$.

For the rod 3, we perform calculations in the same way as for the rod 1. As a result, we obtain:

$$
\begin{aligned}
& d \boldsymbol{w}^{(3)}(s)=\boldsymbol{w}_{M}^{(3)}(s) d \boldsymbol{M}_{B}+\boldsymbol{w}_{R}^{(3)}(s) d \boldsymbol{R}_{B}, \\
& d \boldsymbol{M}^{(3)}(s)=\boldsymbol{M}_{M}^{(3)}(s) d \boldsymbol{M}_{B}+\boldsymbol{M}_{R}^{(3)}(s) d \boldsymbol{R}_{B}, \\
& d \boldsymbol{Q}^{(3)}(s)=\boldsymbol{Q}_{M}^{(3)}(s) d \boldsymbol{M}_{B}+\boldsymbol{Q}_{R}^{(3)}(s) d \boldsymbol{R}_{B},
\end{aligned}
$$

where $\quad \boldsymbol{w}_{M}^{(3)}(s)=\boldsymbol{A}^{(3)}(s) \tilde{\boldsymbol{E}}^{(3)}(0), \quad \boldsymbol{w}_{R}^{(3)}(s)=\boldsymbol{A}^{(3)}(s) \widetilde{\widetilde{\boldsymbol{G}}}^{(3)}(0)+\boldsymbol{S}^{(3)}(s), \boldsymbol{M}_{M}^{(3)}(s)=\tilde{\boldsymbol{M}}^{(3)}(s) \boldsymbol{w}_{M}^{(3)}(s)$, $\boldsymbol{M}_{R}^{(3)}(s)=\tilde{\boldsymbol{M}}^{(3)}(s) \boldsymbol{w}_{R}^{(3)}(s)+\tilde{\widetilde{\boldsymbol{M}}}^{(3)}(s), \boldsymbol{Q}_{M}^{(3)}(s)=\tilde{\boldsymbol{Q}}^{(3)}(s) \boldsymbol{w}_{M}^{(3)}(s), \boldsymbol{Q}_{R}^{(3)}(s)=\tilde{\boldsymbol{Q}}^{(3)}(s) \boldsymbol{w}_{R}^{(3)}(s)+\tilde{\widetilde{\boldsymbol{Q}}}^{(3)}(s)$.

Let's write the equilibrium equations of the frame in the global coordinate system:

$\boldsymbol{R}_{\boldsymbol{A}}+\boldsymbol{R}_{B}+d \boldsymbol{P}^{[1]}+d \boldsymbol{P}^{[2]}=0, R_{A, 3} l_{2}+M_{A, 1}+M_{B, 1}+l_{2} d P_{3}^{[1]}=0$,

$M_{A, 2}+M_{B, 2}-d P_{3}^{[1]} l_{1}-l_{3} d P_{3}^{[2]}=0,-R_{A, 1} l_{2}+M_{A, 3}+M_{B, 3}-l_{2} d P_{1}^{[1]}-l_{1} d P_{2}^{[1]}-l_{3} d P_{2}^{[2]}=0$.

These equations can be written as follows:

$$
\begin{aligned}
& \boldsymbol{H}^{(1,1)} d \boldsymbol{R}_{A}+\boldsymbol{H}^{(1,2)} d \boldsymbol{M}_{A}+\boldsymbol{H}^{(1,3)} d \boldsymbol{R}_{B}+\boldsymbol{H}^{(1,4)} d \boldsymbol{M}_{B}=d \boldsymbol{J}^{(1)}, \\
& \boldsymbol{H}^{(2,1)} d \boldsymbol{R}_{A}+\boldsymbol{H}^{(2,2)} d \boldsymbol{M}_{A}+\boldsymbol{H}^{(2,3)} d \boldsymbol{R}_{B}+\boldsymbol{H}^{(2,4)} d \boldsymbol{M}_{B}=d \boldsymbol{J}^{(2),},
\end{aligned}
$$

where $\boldsymbol{H}^{(1,1)}=\boldsymbol{I}, \boldsymbol{H}^{(1,2)}=\boldsymbol{0}, \boldsymbol{H}^{(1,3)}=\boldsymbol{I}, \boldsymbol{H}^{(1,4)}=\boldsymbol{0}, \boldsymbol{H}^{(2,1)}=\left[\begin{array}{ccc}0 & 0 & l_{2} \\ 0 & 0 & 0 \\ -l_{2} & 0 & 0\end{array}\right], \boldsymbol{H}^{(2,2)}=\boldsymbol{I}, \boldsymbol{H}^{(2,3)}=\boldsymbol{0}$,

$\boldsymbol{H}^{(2,4)}=\boldsymbol{I}, d \boldsymbol{J}^{(1)}=-d \boldsymbol{P}^{[1]}-d \boldsymbol{P}^{[2]}, d \boldsymbol{J}^{(2)}=\left[\begin{array}{l}-l_{2} d P_{3}^{[1]} \\ l_{1} d P_{3}^{[1]}+l_{3} d P_{3}^{[2]} \\ l_{2} d P_{1}^{[1]}+l_{1} d P_{2}^{[1]}+l_{3} d P_{2}^{[2]}\end{array}\right]$.

( $\boldsymbol{I}$ and $\boldsymbol{O}$ are unit and zero third-order matrices). Given the direction of the axes $x_{j}^{(2)}$ and $x_{j}^{(3)}(j=1,2,3)$, we can write the following equalities:

$$
\begin{aligned}
& d w_{1}^{(2)}\left(l_{2}\right)=d x_{2}^{(2)}\left(l_{2}\right)=d x_{1}^{(3)}\left(l_{3}\right)=d w_{9}^{(3)}\left(l_{3}\right), \\
& d w_{2}^{(2)}\left(l_{2}\right)=d x_{3}^{(2)}\left(l_{2}\right)=d x_{3}^{(3)}\left(l_{3}\right)=d w_{2}^{(3)}\left(l_{3}\right) \\
& d w_{3}^{(2)}\left(l_{2}\right)=\left(d x_{2}^{(2)}\right)^{\prime}\left(l_{2}\right)=d \phi^{(2)}\left(l_{2}\right)=d \phi^{(3)}\left(l_{3}\right)=\left(d x_{2}^{(3)}\right)^{\prime}\left(l_{3}\right)=d w_{3}^{(3)}\left(l_{3}\right), \\
& d w_{4}^{(2)}\left(l_{2}\right)=\left(d x_{3}^{(2)}\right)^{\prime}\left(l_{2}\right)=-d \psi^{(2)}\left(l_{2}\right)=-d \theta^{(3)}\left(l_{3}\right)=-d w_{5}^{(3)}\left(l_{3}\right), \\
& d w_{5}^{(2)}\left(l_{2}\right)=d \theta^{(2)}\left(l_{2}\right)=-d \psi^{(3)}\left(l_{3}\right)=\left(d x_{3}^{(1)}\right)^{\prime}\left(l_{3}\right)=d w_{4}^{(1)}\left(l_{3}\right), \\
& d w_{9}^{(2)}\left(l_{2}\right)=d x_{1}^{(2)}\left(l_{2}\right)=-d x_{2}^{(3)}\left(l_{3}\right)=-d w_{1}^{(3)}\left(l_{3}\right) .
\end{aligned}
$$

These equalities can be represented as follows:

$$
\boldsymbol{N} d \boldsymbol{w}^{(2)}\left(l_{2}\right)=\boldsymbol{T}^{[2]} d \boldsymbol{w}^{(3)}\left(l_{3}\right),
$$

where $\boldsymbol{T}^{[2]}$ and $\boldsymbol{N}$ are matrices of order $6 \times 9$ for which $\boldsymbol{T}_{1,9}^{[2]}=\boldsymbol{T}_{2,2}^{[2]}=\boldsymbol{T}_{3,3}^{[2]}=\boldsymbol{T}_{5,4}^{[2]}=1, \boldsymbol{T}_{4,5}^{[2]}=\boldsymbol{T}_{9,1}^{[2]}=$ 
$=-1, N_{i, i}=1(i=1 \div 5,9)$, and the rest of the elements are zero.

Let's use relations (19) for $s=l_{2}$ and (23) for $s=l_{3}$ :

$$
\begin{aligned}
& d \boldsymbol{w}^{(2)}\left(l_{2}\right)=\boldsymbol{w}_{M}^{(2)}\left(l_{2}\right) d \boldsymbol{M}_{A}+\boldsymbol{w}_{R}^{(2)}\left(l_{2}\right) d \boldsymbol{R}_{A}+\boldsymbol{w}_{P}^{(2)}\left(l_{2}\right) d \boldsymbol{P}^{[1]}, \\
& d \boldsymbol{w}^{(3)}\left(l_{3}\right)=\boldsymbol{w}_{M}^{(3)}\left(l_{3}\right) d \boldsymbol{M}_{B}+\boldsymbol{w}_{R}^{(3)}\left(l_{3}\right) d \boldsymbol{R}_{B} .
\end{aligned}
$$

Then (24) can be written as follows:

$$
\boldsymbol{H}^{(3,1)} d \boldsymbol{R}_{A}+\boldsymbol{H}^{(3,2)} d \boldsymbol{M}_{A}+\boldsymbol{H}^{(3,3)} d \boldsymbol{R}_{B}+\boldsymbol{H}^{(3,4)} d \boldsymbol{M}_{B}=d \boldsymbol{J}^{(3)},
$$

where $\boldsymbol{H}^{(3,1)}=\boldsymbol{N} \boldsymbol{w}_{R}^{(2)}\left(l_{2}\right), \boldsymbol{H}^{(3,2)}=\boldsymbol{N} \boldsymbol{w}_{M}^{(2)}\left(l_{2}\right), \boldsymbol{H}^{(3,3)}=-\boldsymbol{T}^{[2]} \boldsymbol{w}_{R}^{(3)}\left(l_{3}\right), \boldsymbol{H}^{(3,4)}=-\boldsymbol{T}^{[2]} \boldsymbol{w}_{M}^{(3)}\left(l_{3}\right)$, $d \boldsymbol{J}^{(3)}=-\boldsymbol{N} \boldsymbol{w}_{P}^{(2)}\left(l_{2}\right) d \boldsymbol{P}^{[1]}$.

The system of matrix equations (2) and (25) can be written as a single matrix equation:

$$
\boldsymbol{H} d \boldsymbol{R}=d \boldsymbol{J},
$$

$$
\boldsymbol{H}=\left[\begin{array}{llll}
\boldsymbol{H}^{(1,1)} & \boldsymbol{H}^{(1,2)} & \boldsymbol{H}^{(1,3)} & \boldsymbol{H}^{(1,4)} \\
\boldsymbol{H}^{(2,1)} & \boldsymbol{H}^{(2,2)} & \boldsymbol{H}^{(2,3)} & \boldsymbol{H}^{(2,4)} \\
\boldsymbol{H}^{(3,1)} & \boldsymbol{H}^{(3,2)} & \boldsymbol{H}^{(3,3)} & \boldsymbol{H}^{(3,4)}
\end{array}\right], d \boldsymbol{R}=\left[\begin{array}{l}
d \boldsymbol{R}_{A} \\
d \boldsymbol{M}_{A} \\
d \boldsymbol{R}_{B} \\
d \boldsymbol{M}_{B}
\end{array}\right], d \boldsymbol{J}=\left[\begin{array}{l}
d \boldsymbol{J}^{(1)} \\
d \boldsymbol{J}^{(2)} \\
d \boldsymbol{J}^{(3)}
\end{array}\right] .
$$

Solving equation (29), we find the vector $d \boldsymbol{R}=\boldsymbol{H}^{-1} d \boldsymbol{J}$, i.e. determine the increments of support reactions $d \boldsymbol{R}_{A}, d \boldsymbol{M}_{A}, d \boldsymbol{R}_{B}, d \boldsymbol{M}_{B}$, which allows using formulas (13), (25) and (28) to determine the increments of the point displacements of the frame rods curved axes at this calculation step $\boldsymbol{H}$.

Remark. It should be noted that the dimension of the matrix (in other words, the dimension of the system of equations represented in the form of matrix equality (29)) is equal to the dimension of the column matrix $\boldsymbol{d} \boldsymbol{R}$, i.e., the degree of static indeterminacy of the frame, which is many times less than the number of unknowns using the finite method elements.

Example. П-shaped reinforced concrete frame (Fig. 1) is under the action of two constant forces $\boldsymbol{P}_{1}^{[1]}$ and $\boldsymbol{P}_{1}^{[2]}$ (gravity forces of two loads $M_{1}$ and $M_{2}$ located in the nodes of the frame) and three variable forces $\boldsymbol{P}_{2}^{[1]}, \quad \boldsymbol{P}_{3}^{[1]}$ and $\boldsymbol{P}_{3}^{[2]}$, the values of which change over time as follows: $P_{2}^{[1]}=10\left(1+\sin 2 \pi \frac{t+T_{2} / 4}{T_{2}}\right) k N, \quad P_{3}^{[2]}=10\left(1+\sin 2 \pi \frac{t+T_{3} / 4}{T_{3}}\right) \quad\left(T_{2}=6 s, T_{3}=4 \mathrm{~s}\right)$. Load masses $m_{1}=m_{2}=4000 \mathrm{~kg}$. Rod lengths $l_{1}=l_{3}=12 \mathrm{~m}, l_{2}=10 \mathrm{~m}$, cross section dimensions $h=0,4 \mathrm{~m}$, $b=0,5 \mathrm{~m}$, concrete class C16/20. Reinforcement is twelve rods with a diameter $10 \mathrm{~mm}$, located symmetrically relative to the local axes $x_{2}^{(j)}$ and $x_{3}^{(j)}$ (j is a rod number), material is A400C steel. It is necessary to determine the motion of loads.

Since the periods of force changes are large enough, the motion of the frame can be considered quasistatic. As a result of using the algorithm above, the motion of loads was determined. Figures 3 and 4 show graphs of changes in the coordinates $x_{2}$ and $x_{3}$ of masses 1 and 2 over time ( 1 for the coordinate $x_{2}, 2$ for $\left.x_{3}\right)$.

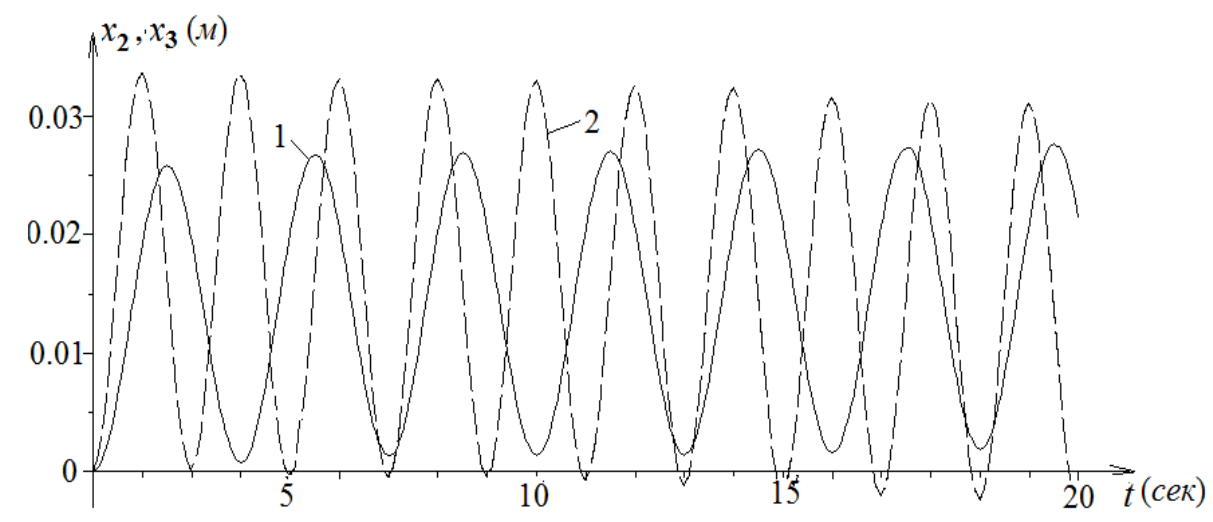

Fig. 3. Graphs of $x_{2}$ and $x_{3}$ load coordinates changes $K_{1}$ 


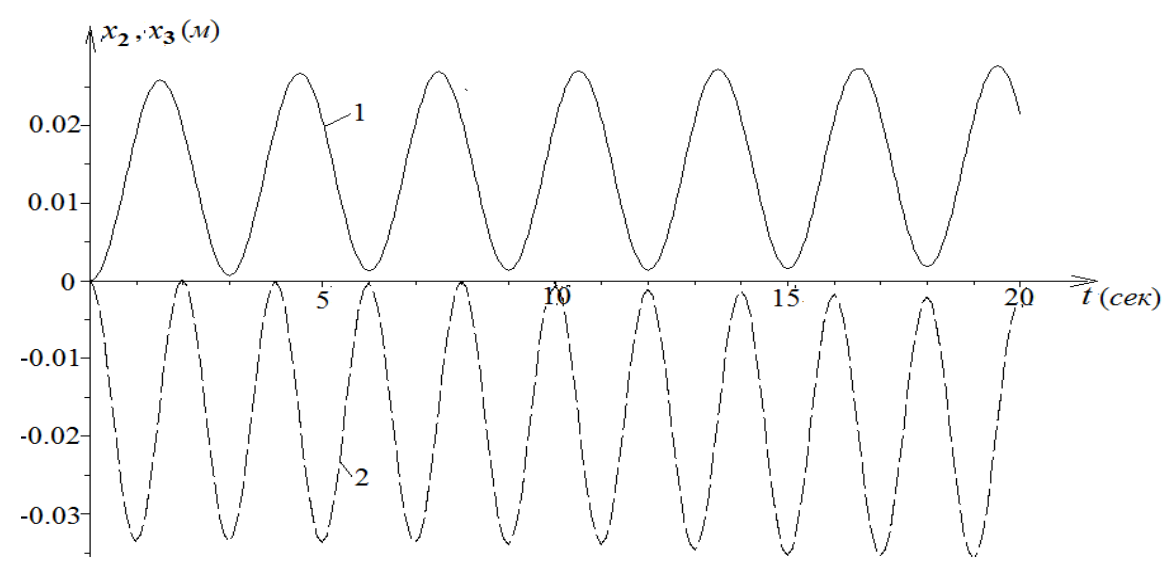

Fig. 4. Graphs of changes in coordinates $x_{2}$ and $x_{3}$ of the load $K_{2}$

Research results. Based on the method for constructing differential equations of spatial nonlinear elastoplastic bending of reinforced concrete beams and the algorithm for applying the boundary element method for solving nonlinear problems proposed by the authors, a method for solving quasistatic problems for reinforced concrete frames is developed.

Conclusions. The developed method is the basis for constructing a dynamic calculation of reinforced concrete frame structures necessary for the design of structures taking into account seismic effects. At the same time, the resolving system of equations has a dimension many times smaller than the dimensions of a similar system when using the finite element method because of the need to split the columns and beams of the structure into a very large number of finite elements due to the fact that the deformation properties of concrete are different in tension and compression zones, as well as in loading and unloading areas. This greatly simplifies and refines the calculation and analysis of its results.

\section{References}

[1] A.S. Gorodetsky, U.D. Geraimovich, "Pryamoy dinamicheskiy raschet zdaniy na seysmicheskie vozdeystviya $\mathrm{s}$ uchetom fizicheski nelineynoy raboti materiala konstrukciy", Bubivelni construkcii, vol. 64, pp. 80-87, 2006.

[2] A.S. Gorodetsky, U.D. Geraimovich, V.S. Shmucler, "Metodi ocenki rabotosposobnosti konstrukcii pri dinamicheskih vozdeystviyah", Naukoviy visnik budivnitstva, vol. 43, pp. 62-71, 2007.

[3] M.A. Mardjanishvili, L.M. Mardjanishvili, S.M. Mardjanishvili, Sovremenie seysmostoykie zdaniya I metodi rascheta. Tbilisi.: Izdatelstvo Tbiliskogo gos. universiteta, 2002.

[4] U.I. Nemchinov, N.G. Marienkov, "Raschet kolebaniy zdaniy I prochnosti gelezobetonih sten na zadanie akselerogrami", Vivnik Odeskoi dergavnoi akademii budivnictva ta arhitekturi, vol. 49, pp. 15-25, 2013.

[5] A.V. Perelmuter, V.I. Slivker, Raschetnie modeli soorugeniy i vozmognost ih analiza. Kiev.: Stal, 2002.

[6] K. Rakech Goel. "Evaluation of nonlinear static procedures using strong-motion building records", SMIP04, Seminar proceeding, 2005, pp. 70-81.

[7] V.M. Fomin, "Primenenie metoda granichnih elementov pri Isledovanii kvaziststicheskih zadach dlya gelezobetonih balok i ram s uchetom fizicheskoy i geometricheskoy nelineynostey i plastichnosti betona", Visnik KNUTD, no. 4 (112), pp. 11-18, 2017.

[8] V.M. Fomin, "Postroenie differencialnih uravneniy prostranstvenogo izgiba gelezobetonih balok i ram s uchetom fizicheskoy i geometricheskoy nelineynostey i plastichnosti betona", Visnik KNUTD, no. 1 (106), pp. 43-50, 2017. 


\title{
ДОСЛІДЖЕННЯ ПРОСТОРОВОГО КВАЗІСТАТИЧНОГО ЗГИНУ

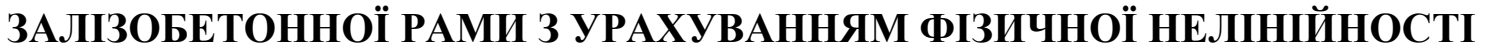 І ПЛАСТИЧНОСТІ БЕТОНУ МЕТОДОМ ГРАНИЧНИХ ЕЛЕМЕНТІВ
}

\author{
${ }^{1}$ Фомін В.М., д.т.н., професор, \\ vmfomin39@gmail.com, ORCID: 0000-0003-4814-6415 \\ ${ }_{1}^{1}$ Фоміна І.П., ст. викладач, \\ fomina@ogasa.org.ua, ORCID: 0000-0001-7366-1537 \\ ${ }^{1}$ Одеська державна академія будівництва та архітектури \\ вул. Дідріхсона, 4, м. Одеса, 65029, Україна
}

\begin{abstract}
Анотація. Виконання прямого динамічного розрахунку просторових рамних залізобетонних конструкцій з урахуванням нелінійної роботи і пластичних властивостей матеріалів $\epsilon$ досить складним завданням. При проведенні подібного розрахунку методом кінцевих елементів виникають труднощі на обчислювальному етапі і при аналізі результатів у зв'язку з тим, що розмірність системи рівнянь цього методу дуже велика, так як потрібно розбиття елементів залізобетонних рам на велику кількість дрібних кінцевих елементів через відмінності деформаційних властивостей бетону в стислій і розтягнутій зонах, а також в стадіях навантаження і розвантаження.

У зв'язку з вищесказаним виникає необхідність в застосуванні альтернативних методів, особливо при дослідженні багатоциклових динамічних процесів, наприклад, при сейсмічних впливах.

На основі методики побудови диференціальних рівнянь просторового нелінійнопружного вигину залізобетонних балок і алгоритму застосування методу граничних елементів при вирішенні нелінійних задач, запропонованих автором, розробляється алгоритм вирішення квазістатичних задач для залізобетонних рам.
\end{abstract}

На кожному зі стрижнів вибирається локальна система координат. У цій системі для кожного зі стрижнів записується система диференціальних рівнянь просторового вигину в приростах координат центру поперечного перерізу і кута кручення з урахуванням фізичної та геометричної нелінійностей і пластичності бетону. На підставі алгоритму, запропонованого автором, за допомогою використання фундаментального рішення задачі Коші для зазначеної системи для кожного стержня можуть бути побудовані матриці, за допомогою яких можуть бути знайдені вектори приростів переміщень, згинальних моментів і поперечних сил в кожному з поперечних перерізів стрижня.

Системи матричних рівнянь, що визначають ці співвідношення можна записати для всієї рами у вигляді єдиного матричного рівняння.

На основі вирішення цих рівнянь визначається приріст напружень в стержнях рами і зміни їх зігнутих осей на кожному кроці розрахунку.

Ключові слова: метод граничних елементів, залізобетонні балки і рами, пропросторовий вигин, фізична нелінійність і пластичність бетону. 


\title{
ИССЛЕДОВАНИЕ ПРОСТРАНСТВЕННОГО КВАЗИСТАТИЧЕСКОГО ИЗГИБА ЖЕЛЕЗОБЕТОННОЙ РАМЫ С УЧЕТОМ ФИЗИЧЕСКОЙ НЕЛИНЕЙНОСТИ И ПЛАСТИЧНОСТИ БЕТОНА МЕТОДОМ ГРАНИЧНЫХ ЭЛЕМЕНТОВ
}

\author{
'Фомин В.М., д.т.н., профессор, \\ vmfomin39@gmail.com, ORCID: 0000-0003-4814-6415 \\ ${ }_{1}^{1}$ Фомина И.П., ст. преподаватель, \\ fomina@ogasa.org.ua, ORCID: 0000-0001-7366-1537 \\ ${ }^{1}$ Одесская государственная академия строительства и архитектуры \\ ул. Дидрихсона, 4, г. Одесса, 65029, Украина
}

Аннотация. Выполнение прямого динамического расчета пространственных рамных железобетонных конструкций с учетом нелинейной работы и пластических свойств является достаточно сложной задачей. При проведении подобного расчета методом конечных элементов возникают трудности на вычислительном этапе и при анализе результатов в связи с тем, что размерность системы уравнений этого метода очень велика, так как необходимо разбиение элементов железобетонных рам на большое количество мелких конечных элементов изза различий где-формационных свойств бетона в сжатой и растянутой зонах, а также в стадиях погрузки и разгрузки.

В связи с вышесказанным возникает необходимость в применении альтернативных методов, особенно при исследовании многоцикловых динамических процессов, например, при сейсмических воздействиях.

На основе методики построения дифференциальных уравнений пространственного нелинейно-упругого изгиба железобетонных балок и алгоритма применения метода граничных элементов при решении нелинейных задач, предложенных автором, разрабатывается алгоритм решения квазистатических задач для железобетонных рам.

На каждом из стержней выбирается локальная система координат. В этой системе для каждого из стержней записывается система дифференциальных уравнений пространственного изгиба в приращенных координат центра поперечного сечения и угла кручения с учетом физической и геометрической нелинейностей и пластичности бетона. На основании алгоритма, предложенного автором, посредством использования фундаментального решения задачи Коши для указанной системы для каждого стержня могут быть построены матрицы, с помощью которых могут быть найдены векторы приращений перемещений, изгибающих моментов и поперечных сил в каждом из поперечных сечений стержня.

Системы матричных уравнений, определяющих эти соотношения, можно записать для всей рамы в виде единого матричного уравнения.

На основе решения этих уравнений определяется приращения напряжений в стержнях рамы и изменения их изогнутых осей на каждом шаге расчета.

Ключевые слова: метод граничных элементов, железобетонные балки и рамы, пространственный изгиб, физическая нелинейность и пластичность бетона.

Стаття надійшла до редакції 25.02.2020 